\title{
Construction of permanently inducible miRNA- based expression vectors using site-specific recombinases
}

\author{
Sara E Garwick-Coppens ${ }^{1}$, Adam Herman ${ }^{2}$ and Scott Q Harper ${ }^{1,3^{*}}$
}

\begin{abstract}
Background: RNA interference (RNAi) is a conserved gene silencing mechanism mediated by small inhibitory microRNAs (miRNAs).

Promoter-driven miRNA expression vectors have emerged as important tools for delivering natural or artificially designed miRNAs to eukaryotic cells and organisms. Such systems can be used to query the normal or pathogenic functions of natural miRNAs or messenger RNAs, or to therapeutically silence disease genes.

Results: As with any molecular cloning procedure, building miRNA-based expression constructs requires a time investment and some molecular biology skills. To improve efficiency and accelerate the construction process, we developed a method to rapidly generate miRNA expression vectors using recombinases instead of more traditional cut-and-paste molecular cloning techniques. In addition to streamlining the construction process, our cloning strategy provides vectors with added versatility. In our system, miRNAs can be constitutively expressed from the U6 promoter, or inducibly expressed by Cre recombinase. We also engineered a built-in mechanism to destroy the vector with Flp recombinase, if desired. Finally, to further simplify the construction process, we developed a software package that automates the prediction and design of optimal miRNA sequences using our system.

Conclusions: We designed and tested a modular system to rapidly clone miRNA expression cassettes. Our strategy reduces the hands-on time required to successfully generate effective constructs, and can be implemented in labs with minimal molecular cloning expertise. This versatile system provides options that permit constitutive or inducible miRNA expression, depending upon the needs of the end user. As such, it has utility for basic or translational applications.
\end{abstract}

\section{Background}

The central dogma of molecular biology describes how the genetic information encoded in DNA is used to make proteins. Although RNAs were long known to play crucial roles in this process (e.g. mRNAs, tRNAs, rRNAs), their importance in gene expression control has dramatically expanded with the recent discovery of RNA interference (RNAi) [1,2]. In the simplest terms, RNAi refers to sequence-specific gene silencing occurring after mRNA transcription, and the principal regulators of this process are small, non-coding microRNAs (miRNAs) $[3,4]$.

\footnotetext{
* Correspondence: scott.harper@nationwidechildrens.org

'Center for Gene Therapy, The Research Institute at Nationwide Children's

Hospital, Columbus, Ohio, 43205, USA

Full list of author information is available at the end of the article
}

Natural miRNAs are encoded in the genomes of eukaryotes and some viruses [2-7]. Over 1,000 miRNAs were identified in recent years, and the major steps and players involved in miRNA biogenesis have been largely defined [8-24]. This knowledge provided the foundation for developing expression systems to deliver engineered inhibitory RNAs, like shRNAs and artificial miRNAs, to various cells and tissues in vitro and in vivo [9,25-28]. In essence, these vectors operate as siRNA or mature miRNA delivery vehicles; as there is not universal nomenclature for such systems, we note that they are referred to as "artificial miRNA shuttles" in this manuscript. Importantly, these artificial miRNA shuttles can be engineered to knockdown any gene of interest $[9,25,26,28,29]$. As a result, they have emerged as powerful molecular tools for querying natural miRNA

\section{Ciomed Central}


function, delivering inhibitory RNAs for functional genomics studies, or developing RNAi-based therapies for disease [30]. Indeed, several pre-clinical studies support that RNAi-based therapies are promising approaches for treating dominant genetic diseases, viral pathogens, and cancer [30-40].

Our laboratory is interested in developing RNAi therapies for dominantly inherited disorders $[34,40]$. This work requires delivering artificial therapeutic miRNA shuttles to target cells and tissues. Numerous groups, including ours, have described various expression vectors in recent years [25-28,32,33,36,41-51]. The most commonly used systems rely upon constitutively active promoters, such as U6, to drive inhibitory RNA transcription. These vectors are typically constructed using traditional cut-and-paste molecular cloning techniques, and multiple constructs are often required to identify ones capable of effectively silencing target genes [29]. To streamline the construction process and make it less labor-intensive, we developed a procedure to rapidly generate artificial miRNA expression vectors using a commercially available phage recombinase system (Gateway ${ }^{\circledR}$ Technology from Invitrogen ${ }^{\mathrm{TM}}$ ). In so doing, we also created a method that provides tightly controlled, inducible miRNA expression, and a built-in mechanism to destroy the vector, if desired. We named our method the Gateway ${ }^{\circledR}$-Ready Inducible MiRNA (GRIM) expression system (Figure 1). In this manuscript, we describe a detailed protocol for GRIM vector construction and demonstrate the functionality of our system in mammalian cells.

\section{Methods \\ GRIM Destination Vector construction}

The GRIM destination vector was constructed by recombinant PCR from two products. The first product contained the mouse U6 promoter flanked by single FRT and LOXP sites at the 5' and 3' ends, respectively. In addition, 18 nucleotides corresponding to the $5^{\prime}$ portion of the AttP1 site were added downstream of LOXP (product A). Product A primers were: 577 Forward (5' TTCAATTGTAGACTAGTGAAGTTCCTATTCTCTAGAAAG 3') and 685 Reverse (5' GGGGCCCGAGCTTAAGACATAACTTCGTAT AGCATACATTATAC 3 '). The second product (Product B) was the Gateway ${ }^{\circledR}$ Cassette, containing the $\mathrm{ccdB}$ and chloramphenicol resistance $(\mathrm{CmR})$ genes flanked by AttP1 and AttP2 sites, which was amplified from pDONR221. This cassette was also engineered to contain 25 nucleotides of a LoxP site at the 5 ' end. Product B primers were: 684 Forward (5' GTATAATGTATGCTATACGAAGT TATGTCTTAAGCTCGGGCCCC 3') and 578 Reverse (5' AACAATTGGTAACATCAGAGATTTTGAGACAC 3 '). Products A and B were then stitched together by 10 cycles of recombinant PCR using the overlapping LoxP and AttP1 sequences, followed by 30 cycles of exponential amplification using primers 577 and 578. The resultant 2,922 bp PCR product was gel purified, cloned into $\mathrm{pCR}^{\circledR}$-Blunt II-TOPO (Invitrogen), transformed into One Shot ${ }^{\circledR}{ }^{(c d B}$ Survival ${ }^{\mathrm{TM}}$ competent cells (Invitrogen), and plated onto LB agar plates containing chloramphenicol. Minipreps from restriction positive colonies were sequenced to confirm accuracy.

\section{Linear miRNA Donor construction}

The 219 bp linear miRNA donor sequences were constructed from 4 primers, two of which are common to all constructs, and two that are unique to the cloned miRNA sequences. This strategy is described in the Results section and primer sequences detailed in Additional File 1. For this study, we built GRIM vectors containing two different microRNA sequences (miLacZ and miGFP) targeting LacZ and eGFP reporters, respectively (Additional File 1). For miRNA donor construction, one microgram of each primer was mixed together in a 50 microliter reaction with $\mathrm{Pfx}$ polymerase (Invitrogen) and thermocycled using the following conditions: $95^{\circ} \mathrm{C} \times 30 \mathrm{sec}, 45^{\circ} \times 30 \mathrm{sec}$, and $68^{\circ} \mathrm{C} \times$ $30 \mathrm{sec}$ for 35 cycles. Full-length miRNA donor bands were visualized on ethidium bromide stained agarose/TBE gels (Additional File 2) to ensure primer extension. However, we found that gel or column purification was unnecessary for BP cloning into the GRIM destination vector.

\section{$\mathrm{BP}$ recombination reaction and clone verification}

Three microliters of the 50-microliter miRNA donor reaction was combined with $150 \mathrm{ng}$ of the Gateway ${ }^{\circledR}$ Destination vector, and this DNA mixture was then added to a 10 microliter, room temperature BP Clonase reaction (Invitrogen) for 1 hour using manufacturer's instructions. BP Clonase was inactivated with Proteinase $\mathrm{K}$, following manufacturer's instructions, and 2 microliters of the $\mathrm{BP}$ reaction was transformed into chemically competent, ccdB sensitive TOP10 E. coli cells (Invitrogen). Colonies were selected on LB agar plates containing kanamycin. Resistant colonies were DNA miniprepped (Qiagen Spin miniprep kit) and digested with EcoRI (New England Biolabs), and electrophoresed on a $1 \%$ agarose/TBE/ethidium bromide gel to determine clone correctness (Additional File 2). Correctly recombined clones (GRIM.miRNA ${ }^{\text {off }}$ ) show two EcoRI bands of 3,501 and $900 \mathrm{bp}$, while the empty GRIM destination vector has three EcoRI fragments of 3,501, 2,170, and 805 bp (Additional File 2). BP recombination had a $90 \%$ efficiency for both the miLacZ and miGFP constructs. Restriction positive colonies were DNA sequenced (Big Dye ${ }^{\circledR}$ Terminator Cycle Sequencing Kit, Applied Biosystems) using a primer located at the 3' end of the U6 promoter (5' CACAAAAGGA AACTCACCC $\left.3^{\prime}\right)$. 


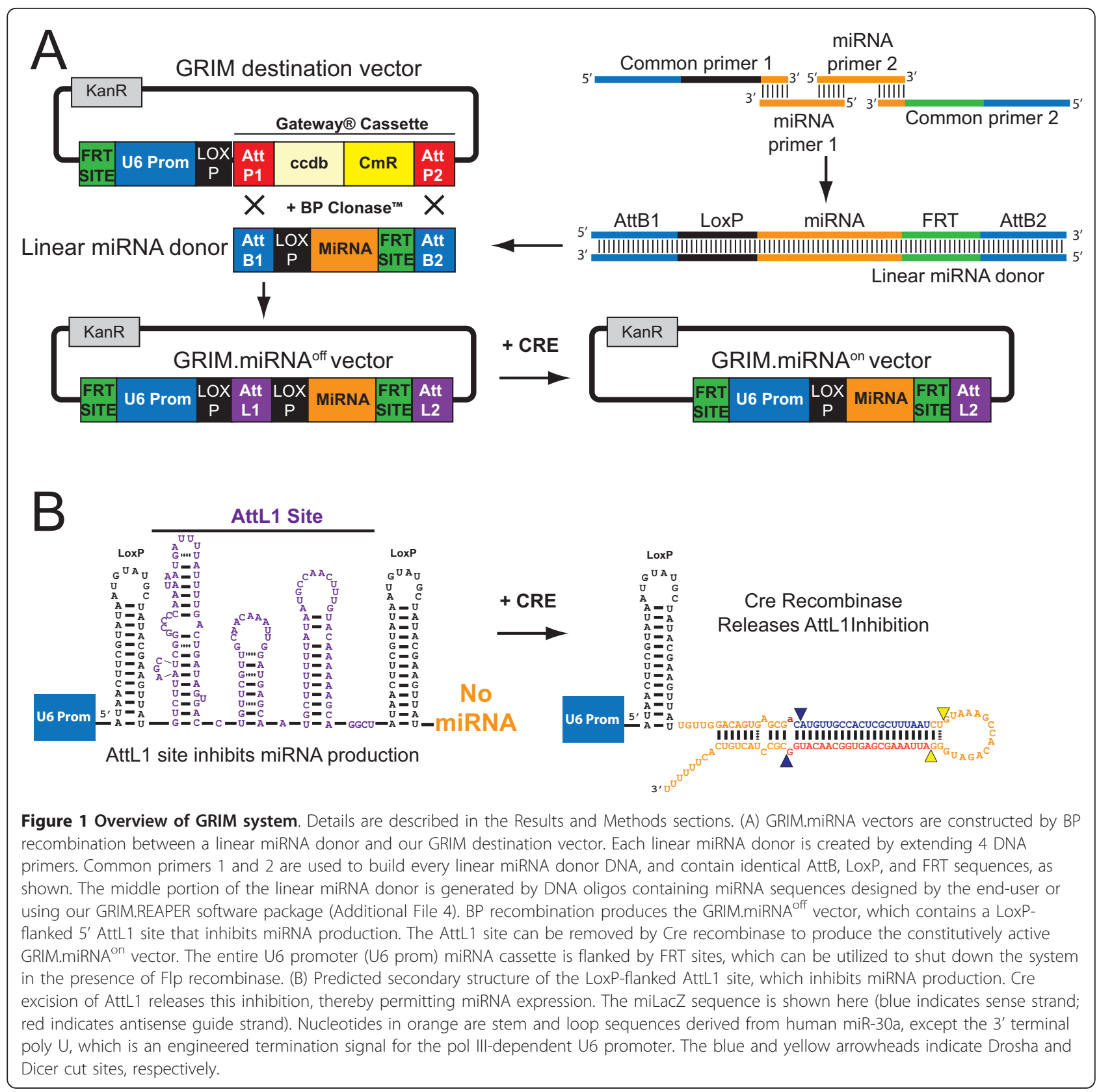

Generating GRIM.miRNA ${ }^{\text {on }}$ vectors in $E$. coli

GRIM.miRNA $^{\text {off }}$ vectors can be permanently switched on by transformation into EL350 E. coli cells, which express Cre recombinase from the arabinose-inducible promoter [52]. Cre recombinase was induced by L-(+)-Arabinose prior to making cells electrocompetent. Cre ${ }^{+}$EL350 cells were then transformed with $1 \mathrm{ng}$ GRIM.miRNA ${ }^{\text {off }}$ plasmid, and grown overnight at $32^{\circ} \mathrm{C}$ on LB agar plates containing kanamycin. Resistant colonies were seeded into miniprep cultures, and agitated overnight in LB/kanamycin liquid media at $32^{\circ} \mathrm{C}$. Following DNA miniprep, FLOXed clones were identified by EcoRI digestion and electrophoresis. Positive GRIM.miRNA ${ }^{\text {on }}$ colonies show two EcoRI bands at 3,501 and $725 \mathrm{bp}$, compared to 3,501 and $900 \mathrm{bp}$ from unFLOXed GRIM.miRNA ${ }^{\text {off }}$ vectors (Additional File 3). In our experiments, Cre excision was $100 \%$ efficient in EL350 cells.

\section{Transient gene silencing studies GRIM.miGFP}

HEK293 cells were co-transfected (Lipofectamine-2000, Invitrogen) with $25 \mathrm{ng}$ of a CMV.eGFP expression vector (pVETLeGFP)[41] and $775 \mathrm{ng}$ indicated miRNA expression plasmid (Figure 2) in duplicate on 24 well 


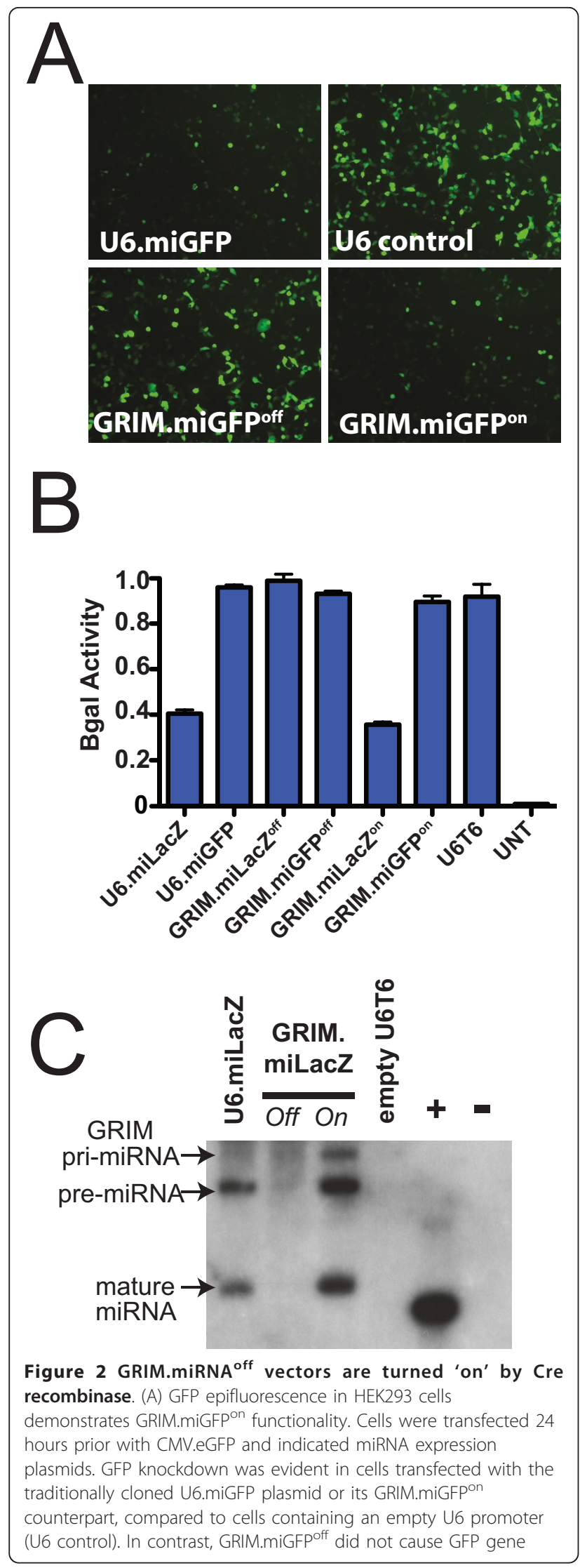

silencing. Images shown are representative of three independent experiments. (B) $\beta$ gal assay confirms the Cre-inducibility of the GRIM system. HEK293 cells were transfected with CMV.LacZ and indicated miRNA or control expression plasmids. Only the traditionally cloned U6.miLacZ and the GRIM.miLacZ ${ }^{\circ}$ analog caused statistically significant LacZ gene silencing (ANOVA, $p<0.05$ ). All other constructs had no impact on $\beta$ gal activity. Error bars indicate standard error of the mean (s.e.m.). U6T6 is an empty U6 promoter plasmid. UNT, untransfected HEK293 cells. (C) Small transcript northern blot shows that Cre induces full-length and processed GRIM.miLacZ. In contrast, GRIM.miLacZ ${ }^{\text {off }}$ vectors do not express fulllength or processed miRNAs. +, indicates a positive control DNA oligo containing the miLacZ antisense sequences. - , indicates negative DNA control corresponding to the miLacZ sense strand. A radiolabeled version of this oligo was also used to probe the blot.

plates. GFP epifluorescence was imaged using a fluorescent inverted microscope equipped with a digital camera 24 hours later.

GRIM.miLacZ. HEK293 cells were co-transfected with 20 ng CMV. $\beta$ gal expression plasmid and $180 \mathrm{ng}$ of the indicated miRNA expression constructs (Figure 2) in triplicate on 96 well plates with white walls and clear bottoms. The next day, cells were lysed for 10 minutes using 10 microliters of Galacto-Light ${ }^{\mathrm{TM}}$ kit lysis buffer (Applied Biosystems). One microliter of lysate was used to quantify total protein by Lowry assay (BioRad DCTM Protein Assay). $\beta$-galactosidase activity was then measured from the remaining 9 microliters of lysate following manufacturer's instructions. Triplicate data were averaged per condition, and three independent experiments performed. $\beta$-galactosidase activity was normalized to Lowry assay-determined protein content. GRIM.milacZ with FIp/FRT system

HEK293 cells were co-transfected with 10 ng CMV. $\beta$ gal and indicated plasmids (Figure $3 \mathrm{~B}$ ). Where indicated, cells received $30 \mathrm{ng}$ CMV.Cre, 130 ng GRIM.miLacZoff, and/or $30 \mathrm{ng}$ CMV.Flp recombinase. $\beta$ gal activity was measured as described above. All wells received equivalent molar amounts of plasmid, such that an empty U6 plasmid was used to normalize DNA loading in transfection liposomes, when necessary.

\section{Northern blot}

Four micrograms of indicated GRIM.miLacZ and control plasmids were transfected into HEK293 cells on 6 well plates. One day later, total RNA was extracted using the miRVANA kit (Ambion). Five micrograms of total RNA per well was loaded on an urea/polyacrylamide/TBE gel, along with DNA oligonucleotides containing antisense $(+)$ or sense (-) miLacZ sequences, as previously described [33]. The gel was run at $20 \mathrm{~mA}$ until bromophenol blue dye reached the bottom, then separated RNAs were transferred to Nylon membranes (Nytran $\mathrm{N}+$ ) at $200 \mathrm{~mA}$ for 45 minutes in $0.5 \times$ TBE. Membranes were then cross- 


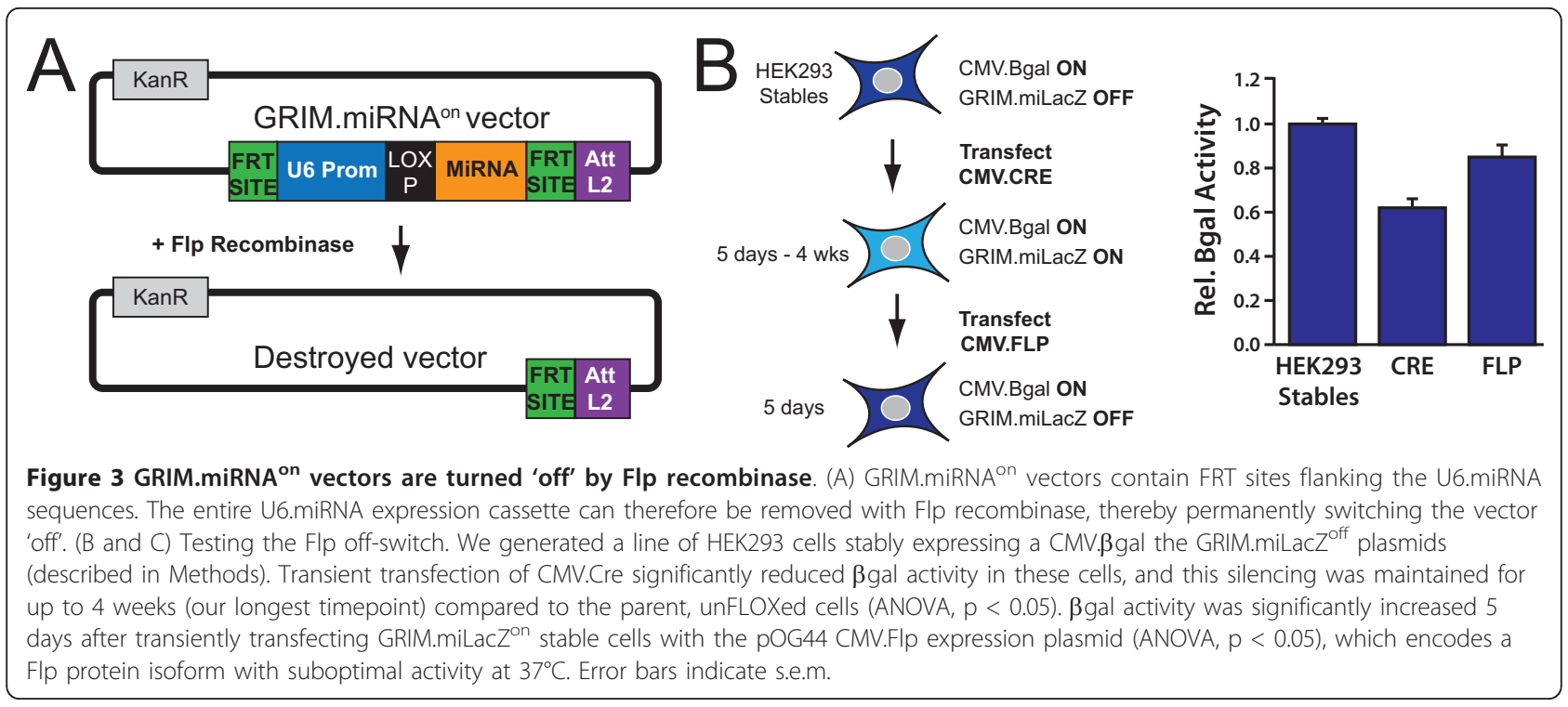

linked and hybridized with a ${ }^{32} \mathrm{P}$ end-labelled DNA oligo (5'-End Labelling Kit, GE Healthcare) that detected the antisense guide strand of the miLac $Z$ sequence, in Oligo Hyb Buffer (Ambion) for two days at $36^{\circ} \mathrm{C}$. The membrane was then washed three times at room temperature and twice at $36^{\circ} \mathrm{C}$ in $2 \times \mathrm{SSC}$, exposed to autoradiographic film (Amersham Hyperfilm ${ }^{\mathrm{TM}}$, GE Healthcare) at $-80^{\circ} \mathrm{C}$, and developed 7 days later.

\section{Generation of Stable HEK293 cells}

HEK293 cells were transfected (Lipofectamine-2000, Invitrogen) with a 1:3 molar ratio of CMV. $\beta$ gal and PGK.Neo plasmids in a $60 \mathrm{~mm}$ plate. Two days later, $400 \mu \mathrm{g} / \mathrm{ml} \mathrm{G} 418$ was added to tissue culture media (DMEM with $10 \%$ FBS, 1\% penicillin/streptomycin, 1\% L-glutamine) and cells were passaged and selected for 4 weeks. G418 resistant cells were then plated at low density, 24 different single colonies were picked and expanded, and representative cells from each colony were stained for $\beta$-gal activity ( $\beta$-gal Staining Kit, Invitrogen) using manufacturer's instructions. Six of 24 colonies were $\beta$ gal positive, and we ultimately selected a line that had consistent $\beta$ gal expression in all cells. This line was then co-transfected (Lipofectamine-2000) in a 12-well plate with $1280 \mathrm{ng}$ GRIM.miLacZ ${ }^{\text {off }}$ and $320 \mathrm{ng}$ of a CAG.Puromycin Resistance (PuroR) plasmid. Two days later, we began selection in Puromycin $(1 \mu \mathrm{g} / \mathrm{ml}$ concentration in media) for one month, and then PCR screened resistant cells for GRIM.miLacZ ${ }^{\text {off }}$ presence.

\section{Validation of the FIp/FRT System in Stable HEK293/ßgal cells}

Puro $^{\mathrm{R}} / \mathrm{G}_{418^{\mathrm{R}}}$ stable HEK293/ßgal/GRIM.miLacZ ${ }^{\text {off }}$ cells were transiently transfected with a CMV.Cre expression plasmid (Lipofectamine-2000, Invitrogen), and $\beta$ gal activity was determined in the parent and FLOXed stable cells 5 days later, to ensure GRIM.miLacZmediated $\beta$ gal knockdown. Four weeks later, FLOXed GRIM.miLacZ ${ }^{\text {on }}$ cells were transfected with the pOG44 CMV.Flp expression plasmid (Stratagene). $\beta$ gal activity was then measured 5 days later using the GalactoLight $^{\mathrm{TM}}$ kit lysis buffer (Applied Biosystems), as described above. Data are representative of 3 independent experiments.

\section{Results}

\section{Construction of GRIM vectors: Overview}

Any inhibitory RNA sequence can be cloned into the GRIM vector system using our universal strategy. A general overview of this method is shown in Figure 1 and the detailed protocol is described in Additional Files 1,2 and 3 . The initial cloning reaction requires three major components: the GRIM destination vector, a linear miRNA donor, and BP Clonase ${ }^{\mathrm{TM}}$ enzyme, derived from lambda phage recombinases (Invitrogen ${ }^{\mathrm{TM}}$ ).

The GRIM destination vector contains the RNA polymerase III-dependent mouse U6 promoter cloned upstream of a Gateway ${ }^{\circledR}$ selection cassette. Single FRT and LoxP sites, which are substrates for Flp and Cre recombinases, respectively, flank the U6 promoter at its 5 ' and 3' ends (Figure 1A).

In our system, the linear miRNA donor is based on sequences and structures derived from human miR-30a (Additional File 1) $[9,17,28]$. We build the miRNA donor by annealing 4 DNA primers and filling in gaps with a thermostable DNA polymerase, such as Taq or Pfx. The two outside primers (Figure 1A; common primers 1 and 2) are universal for building all linear 
miRNA donors, while the two internal ones (miRNA primers 1 and 2) provide the unique miRNA sequences designed by the end user (Figure $1 \mathrm{~A}$ and Additional File 1). Common primer 1 contains a terminal AttB1 site followed by a LoxP site and 15 nucleotides derived from the lower stem of human miR-30a (Figure 1 and Additional File 1). Common primer 2 is a minus strand oligonucleotide encoding a terminal AttB2 site, an optional FRT site, 13 nucleotides of the human miR-30a lower stem, and a RNA polymerase III termination signal (TTTTTT). In addition to encoding the unique miRNA sense and antisense sequences, the internal miRNA primers 1 and 2 also contain human miR-30a stem and loop sequences, which provide common complementary regions for primer annealing. The primer design and annealing strategy is detailed in Additional File 1. In addition, we developed a software package that automates the GRIM.miRNA shuttle prediction and design process (Additional File 4).

Extending the two common and two miRNA-specific primers produces a full-length 219 bp linear miRNA donor DNA (Additional Files 1 and 2), which is then used in a BP recombination reaction with the GRIM destination vector (Figure 1). Smaller, incompletely extended products are also generated but lack fulllength AttB sites and are therefore incapable of participating in the BP reaction (Additional File 2). As a result, gel purification of the full-length linear miRNA donor DNA is unnecessary (discussed in greater detail in supplementary materials; Additional Files 1, 2 and 3).

The parent GRIM destination vector is kanamycin resistant (KanR) and contains an active $c c d B$ (control of cell death $B$ ) gene in the Gateway ${ }^{\circledR}$ cassette region. $C c d B$ gene expression is lethal to most transformationcompetent $E$. coli used in laboratories for molecular cloning purposes. BP recombination replaces the $c c d B$ containing Gateway ${ }^{\circledR}$ cassette with the linear miRNA donor DNA. This reaction also converts AttB and AttP sites to a 118 bp hybrid sequence called AttL (Figure 1). Thus, $c c d B$-sensitive cells will only grow when transformed with properly recombined plasmids, which we termed GRIM.miRNA ${ }^{\text {off }}$ vectors.

GRIM.miRNA ${ }^{\text {off }}$ plasmids have KanR and contain a LoxP-flanked AttL1 site located between the mouse U6 promoter and the artificial miRNA shuttle sequence (Figure 1B). This entire cassette is enclosed at both ends by FRT sites. The AttL1 site inhibits miRNA production, thereby conferring inducibility to the system. Mature, functional miRNAs are therefore only produced after the AttL1 site is excised (a.k.a. FLOXed) by Cre recombinase, yielding GRIM.miRNA ${ }^{\text {on }}$ vectors (Figure 1B, Additional File 3). Alternatively, to rapidly generate un-inducible miRNA vectors, GRIM.miRNA ${ }^{\text {off }}$ vectors can be transformed into EL350 E. coli, which express arabinose-inducible Cre recombinase (Additional File 3). Finally, the flanking FRT sites permit irreversible GRIM. miRNA $^{\text {on }}$ shutdown in the presence of Flp recombinase (Figure 1A).

\section{GRIM.miRNA off vectors are turned 'on' by Cre recombinase}

To demonstrate the functionality of our system, we developed two different miRNA donors targeting the $E$. coli LacZ and A. victoria enhanced GFP (eGFP) genes, respectively (Additional File 1, Figure 2). We called these sequences GRIM.miLacZ and GRIM.miGFP. We first tested the gene-silencing efficacy of each construct relative to a comparable U6.miRNA vector prepared using conventional cut-and-paste cloning techniques (U6.miGFP and U6.miLacZ). To do this, we co-transfected HEK293 cells with a CMV.eGFP expression plasmid and U6.miGFP, an empty U6 vector, GRIM. miGFP ${ }^{\text {off }}$, or GRIM.miGFP ${ }^{\text {on }}$ constructs. Fluorescent microscopic imaging 24 hours later revealed distinctly less GFP expression in cells transfected with the U6. miGFP and GRIM.miGFP ${ }^{\text {on }}$ vectors compared to the empty U6 control. In contrast, GFP levels were comparably higher and more widespread in cells expressing the GRIM.miGFP ${ }^{\text {off }}$ plasmid (Figure 2A).

To better quantify gene silencing and confirm that the functionality of our system was not restricted to the miGFP sequence alone, we performed a second gene silencing experiment using LacZ-directed miRNAs (miLacZ) and a $\beta$-galactosidase $(\beta$-gal) reporter assay. Specifically, we transiently co-transfected HEK293 cells with a CMV.LacZ plasmid and conventional U6 or GRIM-driven miLacZ and control expression vectors (Figure 2B). One day later, we determined miLacZdirected gene silencing by measuring $\beta$-gal activity in transfected cells. We found that cells expressing U6. miLacZ and its GRIM.miLacZ ${ }^{\text {on }}$ counterpart had a statistically significant $60 \%$ reduction in $\beta$-gal activity compared to all other controls (Figure $2 \mathrm{~B}$ ). In contrast, the GRIM.miLac $Z^{\text {off }}$ plasmid had no impact on $\beta$-gal levels (Figure 2B).

Our gene silencing results using both GFP and LacZ reporter assays suggested that the unFLOXed GRIM. miLac $Z^{\text {off }}$ vectors were incapable of producing mature, functional miLacZ sequences. To determine this, we performed small transcript northern blots using RNA isolated from HEK293 cells transfected with empty U6 vector, U6.miLacZ, and the FLOXed and unFLOXed GRIM.miLacZ constructs. The original U6.miRNA cassettes and their GRIM.miRNA ${ }^{\text {on }}$ counterparts are identical sequences except the GRIM vectors contain a single LoxP at the 5 ' end of the miRNA shuttle transcript, and are therefore $34 \mathrm{nt}$ longer (Figure $1 \mathrm{~B}$ ). Using a radiolabeled probe targeting the miLac $Z$ antisense strand, we 
detected identical pre-miLacZ and mature miLacZ species in U6.miLacZ and GRIM.miLacZ ${ }^{\text {on }}$ samples, but not in the empty U6 control or, importantly, the GRIM. miLacZ ${ }^{\text {off }}$ lanes (Figure $2 \mathrm{C}$ and Additional File 1). Moreover, the GRIM.miLacZ ${ }^{\text {on }}$ sample had an additional, larger band that migrated at the expected location of the LoxP-containing primary GRIM transcript (Figure 1B and Figure 2C). These results demonstrated that GRIM vectors are tightly regulated by Cre recombinase.

\section{GRIM.miRNA vectors can be turned 'off' by FIp recombinase}

In some cases, it may be desirable to shutdown miRNA expression. For example, over-expressed inhibitory RNAs can sometimes cause unintended, non-specific off-target effects that could be detrimental to the host cell [33,53-55]. Alternatively, strategies for querying normal miRNA function could include measuring specific outcomes in miRNA-expressing cells and then determining how these outcomes change when the miRNA is turned off. We therefore built a mechanism into the GRIM system to permanently remove the FRT-flanked miRNA expression cassette with Flp recombinase (Figure 3).

We hypothesized that the optimal method to demonstrate off-switch functionality was to reverse, rather than prevent, GRIM.miLacZ-mediated $\beta$ gal gene silencing. This strategy required turning 'on' the GRIM.miLac ${ }^{\text {off }}$ vector and allowing sufficient time for $\beta$ gal reduction before shutting down the system with Flp. We hypothesized that this could not be accomplished in the 2-3 day time window provided by a transient transfection experiment. We therefore generated stable HEK293 cell lines expressing G418-selected CMV.LacZ and Puromycin-selected GRIM.miLac $Z^{\text {off }}$ plasmids. After selection, we transfected HEK293 stables with CMV.Cre, cultured cells for several weeks, transfected again with CMV.Flp, and then harvested cells for $\beta$ gal assays. As expected Cre-treated cells (GRIM.miLacZ ${ }^{\text {on }}$ ) had significantly reduced $\beta$ gal activity compared to HEK293 stables expressing GRIM.miLacZ ${ }^{\text {off }}$. Importantly, transient Flp recombinase expression significantly reversed GRIM. miLacZ ${ }^{\text {on }}$ gene silencing (Figure $3 \mathrm{~B}$ ). These results confirmed that the GRIM system could be inactivated by Flp recombinase.

\section{Discussion}

Numerous inhibitory RNA expression vectors have been described in recent years [25,26,28,29,36,42-51]. Firstgeneration vector systems typically relied upon constitutively active, RNA polymerase III-dependent (pol III) promoters, such as $\mathrm{H} 1$ or U6, to drive uncontrolled inhibitory RNA expression [25,29,32]. As the applicability of RNAi as a technology became more apparent, additional expression strategies emerged, including embedding miRNAs into introns or 3' UTRs of translated genes, and using constitutively active or tissue-specific RNA polymerase II-dependent (pol II) promoters to direct transcription $[36,41]$. To achieve even greater control of inhibitory RNA expression, several groups developed doxycycline- or Cre recombinase-inducible shRNA/miRNA systems [42-49]. These inducible vectors have proven useful for studying normal miRNA function, functional genomics, and may also have potential therapeutic applications, by providing fail-safe mechanisms to shut down inhibitory RNA expression if unintended off-target effects arise.

Despite the diversity of expression strategies currently available, virtually all share the common feature that construction requires cut-and-paste molecular techniques, which can be tedious and time-consuming. In addition, developing optimal artificial inhibitory RNAs, such as for therapeutic purposes, often requires building and screening numerous constructs, which adds to the hands-on time needed to ultimately identify the best sequences [29]. Thus, our initial goal for engineering the GRIM system was to simply streamline the construction process by using a procedure dependent upon recombinases instead of restriction enzymes and DNA ligase. We decided to use the Gateway system because it was a proven method, and the enzyme cocktail required to implement the strategy was commercially available (BP Clonase by Invitrogen).

As shown in Figure 1, our GRIM strategy incorporates AttB sites onto the 5' and 3' ends of a linear DNA template for a mir-30-based artificial miRNA shuttle. These AttB sites can then recombine with AttP sites located in the GRIM destination vector, to produce a longer AttL site, which is an AttB/AttP hybrid (BP recombination; Figure $1 \mathrm{~B}$ ). During the conceptual stages of this method, we modelled the GRIM.miRNA ${ }^{\text {off }}$ transcript in silico; based on its sequence and structure, we hypothesized that the AttL site could interfere with miRNA production because of steric hindrance and/or premature transcriptional termination. Specifically, the AttL sequence was predicted to fold into multiple hairpin secondary structures (Figure 1B) that could inhibit transcriptional read-through to the miRNA, and/or impair miRNA processing (steric hindrance); and the AttL DNA template contained three stretches of termination signals for pol III-dependent transcripts ( 4 or more T's; Figure $1 \mathrm{~B}$ and Additional File 5), which could prevent transcription of the full-length primary miRNA transcript (premature termination). From these concerns arose the strategy to FLOX the AttL site and remove it with Cre recombinase. Although we predicted the AttL site would partially interfere with miRNA expression, we were surprised to discover that its presence prevented miRNA production altogether (Figures 1, 2). As a result, 
Cre-inducibility emerged as an important, albeit somewhat unexpected, feature of the GRIM system. Nevertheless, the GRIM system is versatile, and Creinducibility is optional. As previously mentioned, our primary intention was to accelerate the miRNA shuttle construction process, and our initial strategy involved removing the inhibitory AttL site by simple transformation of GRIM.miRNA ${ }^{\text {off }}$ plasmids into Cre-expressing, EL350 E. coli. This virtually foolproof step produces a GRIM.miRNA ${ }^{\text {on }}$ plasmid that is then constitutively 'on' when delivered to mammalian cells.

We also built in a mechanism to excise the GRIM. miRNA $^{\text {on }}$ cassette and turn it 'off' permanently with Flp recombinase, and tested the functionality of this offswitch in stable cell lines (Figure 3). Our goal here was to demonstrate that Flp recombinase could negatively regulate our system. Indeed, transient Flp expression significantly reversed GRIM.miLacZ-mediated silencing, but we noted that $\beta$ gal activity did not return to baseline (Figure 3 ). This was likely because the heat-labile CMV.Flp construct we used (pOG44, Stratagene) has low activity at $37^{\circ} \mathrm{C}$ [56], which was the growth temperature of our HEK293 stables. Indeed, the optimal temperature for pOG44-derived Flp is $30^{\circ} \mathrm{C}$. Exposing GRIM.miLacZ ${ }^{\text {on }}$ stable HEK293 cells to heat-labile Flp for a longer period of time, or expressing a thermostable form of the protein, would further improve the efficiency of gene silencing reversal.

Finally, the current GRIM vector described here relies on the RNA pol III-dependent U6 promoter to drive miRNA shuttle transcription. However, our strategy is likely not limited to pol III promoters alone. Indeed, inserting pol II poly A signals (AATAAA) in two different locations within the current GRIM system would enable the use of tissue-specific pol II promoters (Additional File 5). First, the pol III termination signal (TTTTTT) placed at the 3' end of the artificial miRNA can be replaced by one or more minimal pol II poly A signals (AATAAA). Second, if the putative pol III termination signals in the AttL site inhibit miRNA production via premature termination of the primary transcript, an analogous situation can be produced for a pol II promoter-driven transcript by simply placing one or more AATAAA signals in the GRIM destination vector just downstream of the first LoxP site (Additional File 5). Cre excision would then derepress the pol II-dependent GRIM vector, allowing processive miRNA transcription until correct termination at the downstream polyA signal. As with the pol III-based system described in this manuscript, the option to build in the Flp/FRT shutdown mechanism would also provide the vector with additional versatility. Thus, although the functionality of such a system remains to be tested, building tissue-specific pol II promoters into the GRIM system could further expand the utility of this technology.

\section{Conclusions}

We developed a simple, rapid, and versatile system to generate miRNA expression plasmids using site-specific recombinases. One of the major advantages of our strategy is ease of construction; this feature has benefits for labs with extensive or minimal molecular biology skills (Additional File 6). In addition, our method has a built-in Cre recombinase-dependent 'on' switch that permits tight control of expression, and an optional mechanism to turn expression 'off' using Flp recombinase. Our system can therefore be used for more traditional gene silencing experiments, but also has applications for making inducible transgenic animals or RNAi therapeutics [44,57-60].

\section{Additional material}

Additional file 1: Detailed strategy for designing artificial GRIM miRNAs and the primers required for their construction. Step1. This strategy can be used to express a natural miRNA. However, in this protocol, we describe engineering artificial GRIM miRNAs for knocking down any gene of interest. Each GRIM miRNA is based on hsa-miR-30a sequences and structure. The natural mir-30a mature sequences are replaced by unique sense (blue text) and antisense (red text) sequences derived from the target gene. In this example, we show the miLacZ and miGFP constructs used in this manuscript. The orange nucleotides are derived from human miR-30a, except the $3^{\prime}$ terminal poly $U$, which is added for use as a termination signal for the pol III-dependent U6 promoter. The natural mir-30 Drosha and Dicer cut sites are maintained and indicated by blue and yellow arrowheads, respectively. The mismatch located just upstream of the Drosha cut site (at position -2) should be maintained for proper processing. Choosing effective artificial miRNA sequences targeting a gene of interest still requires some trialand-error empirical testing. However, there are several publications describing specific rules for optimal inhibitory RNA design. We found that incorporating two such guidelines improves the chances of identifying effective artificial miRNA sequences. First, cellular gene silencing proteins (RISC, RNA induced silencing complex) preferentially load the more thermodynamically unstable $5^{\prime}$ end of a miRNA duplex ( e. more AU-rich). Thus, to ensure proper loading of the antisense guide strand, the antisense miRNA strand should have more GC base pairs at its $3^{\prime}$ end than its $5^{\prime}$ end (i.e. the last 3-4 bases on either side; see LacZ and GFP target sites as an example of this requirement). Second, the mature miRNA sequence should have $60 \%$ or less GC content. To simplify design, we built both "rules" into our GRIM.REAPER miRNA design program (Additional File 4). Step 2. The GRIM.miRNA is ultimately transcribed from a DNA template. Step 2 illustrates the specific primer extension strategy used to create the DNA template for a GRIM.miRNA Four primers are annealed and extended using a thermostable DNA polymerase, such as Taq or Pfx. Commons Primers 1 and 2 are used for all GRIM.miRNA constructions. Two unique primers with some common features are used to generate each specific miRNA, as shown in the miLacZ and miGFP examples here. Although the design strategy provided here permits the end-user to create miRNAs and primer sequences manually, we have automated this process using the GRIM. REAPER design program (Additional File 4).

Additional file 2: Generating GRIM.miRNA vectors and screening for positive clones. Step 3. Producing the DNA template for a Gateway Ready miRNA by thermocycling 4 DNA oligos. One microgram each of Common primers 1 and 2, and unique miRNA primers 1 and 2 (generated by the GRIM.REAPER design program), were annealed and extended in a 50 microliter Pfx polymerase reaction (Invitrogen) as follows: $95^{\circ} \mathrm{C} \times 30 \mathrm{sec}, 45^{\circ} \times 30 \mathrm{sec}$, and $68^{\circ} \mathrm{C} \times 30 \mathrm{sec}$ for $35 \mathrm{cycles}$. The full-length $219 \mathrm{bp}$ miRNA donor DNA was visualized on an ethidium bromide stained agarose gel. BP recombination of this product does not require gel or column purification. Step 4: BP Reaction. As described in 
the Methods section, BP recombination results in loss of the $c c d B / C m R$ cassette in the GRIM destination vector. This permits propagation in $c c d B$-sensitive $E$. coli (i.e. most competent cell strains like DH5a or TOP10). Positive colonies are also kanamycin resistant. Correctly recombined GRIM.miRNA ${ }^{\text {off }}$ plasmids have two EcoRI bands of $900 \mathrm{bp}$ and 3,501 bp, as shown. In our hands, 90\% of $c c d B$ negative/kanamycin resistant colonies showed the correct EcoRl restriction pattern. Step 5: DNA sequencing. Plasmids showing the correct EcoRl restriction pattern are DNA sequenced using an oligonucleotide primer located at the $3^{\prime}$ end of the U6 promoter (5' CACAAAAGGAAACTCACCC $3^{\prime}$ ).

Additional file 3: Regulating the GRIM system with Cre or Flp. Step 6. The GRIM.miRNA ${ }^{\text {off }}$ vector is turned 'on' by Cre recombinase excision of the inhibitory AttL1 site. To make a constitutively active vector, AttL1 excision can be accomplished through transformation of the GRIM. miRNA ${ }^{\text {off }}$ vector into EL350 E. Coli, which express Cre recombinase. This 100\% efficient reaction removes $175 \mathrm{bp}$ from the plasmid, such that EcoRI digestion yields 725 bp and 3,501 bp bands, as shown Alternatively, GRIM.miRNA ${ }^{\text {off }}$ vectors can be inducibly turned 'on' in animal cells or tissues expressing Cre recombinase from tissue- or temporal-specific promoters. Step 7. The GRIM.miRNA ${ }^{\text {on }}$ vector can be turned 'off' with Flp recombinase, as described in the Methods.

Additional file 4: GRIM.REAPER miRNA design program. Using the criteria described in Additional File 1, this Java-based software package predicts highly functional artificial miRNAs from gene sequences entered by the end-user. In addition, it outputs the sequence of the DNA oligonucleotides required to build each GRIM.miRNA construct. Instructions: (1) Paste the DNA sequence of the gene of interest into the GRIM.REAPER query box. (2) Save the output text file locally. (3) Open the file. Example output: Query sequence : $1073 \mathrm{nt}$ miRNA position: 70-91 Mature Duplex: 5' CGUUUGGACCCCGAGCCAAACU 3'

|| || || || || || || ||||||| | 3' GAGCAAACCUGGGGCUCGGUUU 5' Full length GRIM. miRNA sequence: 5' UGUUGGACAGUGAG CGAUCGUUUGGACCCCGAGCCAAACUGUAAAGCCACAGAUGGGUUUG GCUCGGGGUCCAAACGAGCGCCUACUGUCACUUUUUUU 3' miRNA primer 1: 5' CCCATCTGTGGCTTTACAGTTTGGCTCGGGGTCCA AACGATCGCTCACTGTCCAAC $3^{\prime}$ miRNA primer $2: 5^{\prime}$ CTGTAAAG CCACAGATGGGTTTGGCTCGGGGTCCAAACGAGCGCCTACTGTCACTTT 3'.

Additional file 5: Model for converting the GRIM system from pol III to pol II dependence. (A) We hypothesize that RNA pol III termination signals (4-6 T's) in the inhibitory AttL1 site cause premature termination of GRIM.miRNAs, which prevents miRNA maturation and ultimately, gene silencing. Cre removes the AttL1-resident poly $\mathrm{T}$ terminators, thereby permitting full-length GRIM miRNA transcription and maturation. (B) Based on this model, the current GRIM system could be used with a pol II-based promoter as well, with some modifications. Specifically, the GRIM destination vector can be modified to contain a pol II responsive poly A signal downstream of the $5^{\prime}$ LoxP site, which would serve to prematurely terminate a full-length GRIM.miRNA. A second poly A signal, downstream of the miRNA cassette, can then be added during the miRNA donor construction. In the unFLOXed state, the upstream poly A signal would be utilized thereby preventing miRNA production and subsequent gene silencing. Cre-mediated excision (FLOXed state) would remove the upstream poly A signal along with the AttL1 site, thereby permitting fulllength miRNA transcription, maturation, and gene silencing

Additional file 6: Flow chart comparing traditional cut-and-paste molecular cloning methods versus the GRIM system. This chart illustrates the streamlined cloning process afforded by the GRIM system, compared to more traditional molecular construction methods. GRIM cloning requires markedly less hands-on and overall time for miRNA expression vector construction. Indeed, correct clones can be generated and confirmed 1-2 days earlier than with traditional molecular methods. Moreover, restriction enzyme and ligation-based methods can sometimes be inefficient and vector generation may require repeated attempts. The high cloning efficiency of the GRIM system ( $90 \%$ ) circumvents this problem.

\section{Acknowledgements}

The authors thank Dr. Jennifer Allen for technical assistance, and members of the Harper Lab for critical reading of this manuscript. This work was supported by a NIH KL2 Clinical and Translational Scholar Award (KL2 RR025752) to SQH, Research Fellowship Grants from the FSH Society (FSHS82010-02 and FSHS-082009-01 to SQH), and startup funds to SQH from the Research Institute at Nationwide Children's Hospital.

\section{Author details}

'Center for Gene Therapy, The Research Institute at Nationwide Children's Hospital, Columbus, Ohio, 43205, USA. Research Information Services, The Research Institute at Nationwide Children's Hospital, Columbus, Ohio, 43205, USA. ${ }^{3}$ Department of Pediatrics, The Ohio State University College of Medicine, Columbus, Ohio, 43205, USA

\section{Authors' contributions}

SQH and SEGC designed the GRIM cloning strategy and wrote the manuscript. SEGC cloned and experimentally tested all vectors. SQH performed northern blots. AH and SQH designed the GRIM.miRNA prediction software package. All authors read and approved the final manuscript.

Received: 12 May 2011 Accepted: 16 November 2011 Published: 16 November 2011

\section{References}

1. Fire A, Xu S, Montgomery MK, Kostas SA, Driver SE, Mello CC: Potent and specific genetic interference by double-stranded RNA in Caenorhabditis elegans. Nature 1998, 391:806-811.

2. Fire AZ: Gene silencing by double-stranded RNA. Cell Death Differ 2007, 14:1998-2012.

3. Bartel DP: MicroRNAs: genomics, biogenesis, mechanism, and function. Cell 2004, 116:281-297.

4. Lai EC: Micro RNAs are complementary to $3^{\prime}$ UTR sequence motifs that mediate negative post-transcriptional regulation. Nat Genet 2002, 30:363-364.

5. Lee RC, Ambros V: An extensive class of small RNAs in Caenorhabditis elegans. Science 2001, 294:862-864.

6. Odling-Smee L: Complex set of RNAs found in simple green algae. Nature 2007, 447:518

7. Grundhoff A, Sullivan CS: Virus-encoded microRNAs. Virology 2011, 411:325-343

8. Cai $\mathrm{X}$, Hagedorn $\mathrm{CH}$, Cullen BR: Human microRNAs are processed from capped, polyadenylated transcripts that can also function as mRNAs. RNA 2004, 10:1957-1966.

9. Zeng Y, Cai X, Cullen BR: Use of RNA polymerase II to transcribe artificial microRNAs. Methods Enzymol 2005, 392:371-380.

10. Tang G: siRNA and miRNA: an insight into RISCs. Trends Biochem Sci 2005, 30:106-114.

11. Borchert GM, Lanier W, Davidson BL: RNA polymerase III transcribes human microRNAs. Nat Struct Mol Biol 2006, 13:1097-1101.

12. Elbashir SM, Harborth J, Lendeckel W, Yalcin A, Weber K, Tuschl T: Duplexes of 21-nucleotide RNAs mediate RNA interference in cultured mammalian cells. Nature 2001, 411:494-498

13. Hutvagner G, McLachlan J, Pasquinelli AE, Balint E, Tuschl T, Zamore PD: A cellular function for the RNA-interference enzyme Dicer in the maturation of the let-7 small temporal RNA. Science 2001, 293:834-838.

14. Hutvagner G, Zamore PD: A microRNA in a multiple-turnover RNAi enzyme complex. Science 2002, 297:2056-2060.

15. Khvorova A, Reynolds A, Jayasena SD: Functional siRNAs and miRNAs exhibit strand bias. Cell 2003, 115:209-216.

16. Lee Y, Ahn C, Han J, Choi H, Kim J, Yim J, Lee J, Provost P, Radmark O, Kim S, Kim VN: The nuclear RNase III Drosha initiates microRNA processing. Nature 2003, 425:415-419.

17. Lee Y, Kim M, Han J, Yeom KH, Lee S, Baek SH, Kim VN: MicroRNA genes are transcribed by RNA polymerase II. Embo J 2004, 23:4051-4060.

18. Macrae IJ, Zhou K, Li F, Repic A, Brooks AN, Cande WZ, Adams PD, Doudna JA: Structural basis for double-stranded RNA processing by Dicer. Science 2006, 311:195-198.

19. Marques JT, Devosse T, Wang D, Zamanian-Daryoush M, Serbinowski $P$, Hartmann R, Fujita T, Behlke MA, Williams BR: A structural basis for 
discriminating between self and nonself double-stranded RNAs in mammalian cells. Nat Biotechnol 2006, 24:559-565.

20. Schwarz DS, Hutvagner G, Du T, Xu Z, Aronin N, Zamore PD: Asymmetry in the assembly of the RNAi enzyme complex. Cell 2003, 115:199-208.

21. Tolia NH, Joshua-Tor L: Slicer and the argonautes. Nat Chem Biol 2007, 3:36-43.

22. Yi R, Qin Y, Macara IG, Cullen BR: Exportin-5 mediates the nuclear export of pre-microRNAs and short hairpin RNAs. Genes Dev 2003, 17:3011-3016.

23. Wang Y, Medvid R, Melton C, Jaenisch R, Blelloch R: DGCR8 is essential for microRNA biogenesis and silencing of embryonic stem cell self-renewal. Nat Genet 2007, 39:380-385.

24. Gregory RI, Yan KP, Amuthan G, Chendrimada T, Doratotaj B, Cooch N, Shiekhattar R: The Microprocessor complex mediates the genesis of microRNAs. Nature 2004, 432:235-240.

25. Paddison PJ, Caudy AA, Bernstein E, Hannon GJ, Conklin DS: Short hairpin RNAs (shRNAs) induce sequence-specific silencing in mammalian cells. Genes Dev 2002, 16:948-958.

26. Zeng Y, Wagner EJ, Cullen BR: Both natural and designed micro RNAs can inhibit the expression of cognate mRNAs when expressed in human cells. Mol Cell 2002, 9:1327-1333.

27. Xia $H$, Mao Q, Paulson HL, Davidson BL: siRNA-mediated gene silencing in vitro and in vivo. Nat Biotechnol 2002, 20:1006-1010.

28. Boudreau RL, Martins I, Davidson BL: Artificial microRNAs as siRNA shuttles: improved safety as compared to shRNAs in vitro and in vivo. Mol Ther 2009, 17:169-175.

29. Harper SQ, Davidson BL: Plasmid-based RNA interference: construction of small-hairpin RNA expression vectors. Methods Mol Biol 2005, 309:219-235.

30. Davidson $B L$, Boudreau RL: RNA interference: a tool for querying nervous system function and an emerging therapy. Neuron 2007, 53:781-788.

31. Gonzalez-Alegre P, Bode N, Davidson BL, Paulson HL: Silencing primary dystonia: lentiviral-mediated RNA interference therapy for DYT1 dystonia. J Neurosci 2005, 25:10502-10509.

32. Harper SQ, Staber PD, He X, Eliason SL, Martins IH, Mao Q, Yang L, Kotin RM, Paulson $\mathrm{HL}$, Davidson BL: RNA interference improves motor and neuropathological abnormalities in a Huntington's disease mouse model. Proc Natl Acad Sci USA 2005, 102:5820-5825.

33. McBride JL, Boudreau RL, Harper SQ, Staber PD, Monteys AM, Martins I, Gilmore BL, Burstein H, Peluso RW, Polisky B, et al: Artificial miRNAs mitigate shRNA-mediated toxicity in the brain: implications for the therapeutic development of RNAi. Proc Natl Acad Sci USA 2008, 105:5868-5873.

34. Wallace LM, Garwick SE, Harper SQ: RNAi Therapy for Dominant Muscular Dystrophies and Other Myopathies. In Muscle Gene Therapy. Edited by: Duan D. New York: Springer; 2010:99-115.

35. Xia H, Mao Q, Eliason SL, Harper SQ, Martins $1 H$, Orr HT, Paulson HL, Yang L, Kotin RM, Davidson BL: RNAi suppresses polyglutamine-induced neurodegeneration in a model of spinocerebellar ataxia. Nat Med 2004, 10:816-820

36. Kota J, Chivukula RR, O'Donnell KA, Wentzel EA, Montgomery CL, Hwang HW, Chang TC, Vivekanandan P, Torbenson M, Clark KR, et al: Therapeutic microRNA delivery suppresses tumorigenesis in a murine liver cancer model. Cell 2009, 137:1005-1017.

37. Lee NS, Dohjima T, Bauer G, Li H, Li MJ, Ehsani A, Salvaterra P, Rossi J: Expression of small interfering RNAs targeted against HIV-1 rev transcripts in human cells. Nat Biotechnol 2002, 20:500-505.

38. Pan Q, Henry SD, Metselaar HJ, Scholte B, Kwekkeboom J, Tilanus HW, Janssen $H \mathrm{~L}$, van der Laan $\mathrm{L}$ : Combined antiviral activity of interferonalpha and RNA interference directed against hepatitis $C$ without affecting vector delivery and gene silencing. J Mol Med 2009, 87:713-722.

39. Rodriguez-Lebron E, Denovan-Wright EM, Nash K, Lewin AS, Mandel RJ: Intrastriatal rAAV-mediated delivery of anti-huntingtin shRNAs induces partial reversal of disease progression in R6/1 Huntington's disease transgenic mice. Mol Ther 2005, 12:618-633.

40. Wallace LM, Harper SQ: RNA interference improves myopathic phenotypes in mice over-expressing FSHD region gene 1 (FRG1). Mol Ther 2011, 19:2048-2054.

41. Harper SQ, Staber PD, Beck CR, Fineberg SK, Stein C, Ochoa D, Davidson BL: Optimization of feline immunodeficiency virus vectors for RNA interference. J Virol 2006, 80:9371-9380.

42. Aagaard L, Amarzguioui M, Sun G, Santos LC, Ehsani A, Prydz H, Rossi JJ: A facile lentiviral vector system for expression of doxycycline-inducible
shRNAs: knockdown of the pre-miRNA processing enzyme Drosha. $\mathrm{Mol}$ Ther 2007, 15:938-945.

43. Qiu L, Wang $H, X i a X$, Zhou $H, X u Z$ : A construct with fluorescent indicators for conditional expression of miRNA. BMC Biotechnol 2008, 8:77.

44. Coumoul X, Shukla V, Li C, Wang RH, Deng CX: Conditional knockdown of Fgfr2 in mice using Cre-LoxP induced RNA interference. Nucleic Acids Res 2005, 33:e102

45. Iovino N, Denti MA, Bozzoni I, Cortese R: A loxP-containing pol II promoter for RNA interference is reversibly regulated by Cre recombinase. RNA Biol 2005, 2:86-92.

46. Yu J, McMahon AP: Reproducible and inducible knockdown of gene expression in mice. Genesis 2006, 44:252-261.

47. Chang HS, Lin CH, Chen YC, Yu WC: Using siRNA technique to generate transgenic animals with spatiotemporal and conditional gene knockdown. Am J Pathol 2004, 165:1535-1541.

48. Tiscornia G, Tergaonkar V, Galimi F, Verma IM: CRE recombinase-inducible RNA interference mediated by lentiviral vectors. Proc Natl Acad Sci USA 2004, 101:7347-7351

49. Meerbrey KL, Hu G, Kessler JD, Roarty K, Li MZ, Fang JE, Herschkowitz Jl, Burrows AE, Ciccia $A$, Sun $T$, et al: The pINDUCER lentiviral toolkit for inducible RNA interference in vitro and in vivo. Proc Natl Acad Sci USA 2011, 108:3665-3670.

50. Fritsch L, Martinez LA, Sekhri R, Naguibneva I, Gerard M, Vandromme M, Schaeffer L, Harel-Bellan A: Conditional gene knock-down by CREdependent short interfering RNAs. EMBO Rep 2004, 5:178-182.

51. van de Wetering M, Oving I, Muncan V, Pon Fong MT, Brantjes H, van Leenen D, Holstege FC, Brummelkamp TR, Agami R, Clevers H: Specific inhibition of gene expression using a stably integrated, inducible smallinterfering-RNA vector. EMBO Rep 2003, 4:609-615.

52. Lee EC, Yu D, Martinez de Velasco J, Tessarollo L, Swing DA, Court DL, Jenkins NA, Copeland NG: A highly efficient Escherichia coli-based chromosome engineering system adapted for recombinogenic targeting and subcloning of BAC DNA. Genomics 2001, 73:56-65.

53. Lu Y, Thomson JM, Wong HY, Hammond SM, Hogan BL: Transgenic overexpression of the microRNA miR-17-92 cluster promotes proliferation and inhibits differentiation of lung epithelial progenitor cells. Dev Biol 2007, 310:442-453.

54. Grimm D, Streetz KL, Jopling CL, Storm TA, Pandey K, Davis CR, Marion P, Salazar F, Kay MA: Fatality in mice due to oversaturation of cellular microRNA/short hairpin RNA pathways. Nature 2006, 441:537-541.

55. Pan Q, de Ruiter PE, von Eije KJ, Smits R, Kwekkeboom J, Tilanus HW, Berkhout B, Janssen HL, van der Laan LJ: Disturbance of the microRNA pathway by commonly used lentiviral shRNA libraries limits the application for screening host factors involved in hepatitis $C$ virus infection. FEBS Lett 2011, 585:1025-1030.

56. Buchholz F, Ringrose L, Angrand PO, Rossi F, Stewart AF: Different thermostabilities of FLP and Cre recombinases: implications for applied site-specific recombination. Nucleic Acids Res 1996, 24:4256-4262.

57. Kleinhammer A, Wurst W, Kuhn R: Gene knockdown in the mouse through RNAi. Methods Enzymol 2010, 477:387-414.

58. Hohenstein P, Slight J, Ozdemir DD, Burn SF, Berry R, Hastie ND: Highefficiency Rosa26 knock-in vector construction for Cre-regulated overexpression and RNAi. PathoGenetics 2008, 1:3.

59. Stern P, Astrof S, Erkeland SJ, Schustak J, Sharp PA, Hynes RO: A system for Cre-regulated RNA interference in vivo. Proc Natl Acad Sci USA 2008, 105:13895-13900.

60. Qiu L, Rivera-Perez JA, Xu Z: A Non-Specific Effect Associated with Conditional Transgene Expression Based on Cre-loxP Strategy in Mice. PLoS One 2011, 6:e18778.

\section{doi:10.1186/1472-6750-11-107}

Cite this article as: Garwick-Coppens et al:: Construction of permanently inducible miRNA-based expression vectors using site-specific recombinases. BMC Biotechnology 2011 11:107. 